\title{
GENERALIZED BLOCKED TRIANGULAR MATRIX RINGS ASSOCIATED WITH FINITE ABELIAN CENTRALIZER NEAR-RINGS
}

\author{
by KIRBY C. SMITH and LEON VAN WYK
}

(Received 2nd April 1996)

\begin{abstract}
For $N$ any member of a large class of finite abelian right centralizer near-rings, the subring of the ring $\operatorname{End}(N)$ of endomorphisms of $(N,+)$ generated by the set of right multiplication maps on $N$ is explicitly described as a generalized blocked triangular matrix ring, which in some cases turns out to be a structural matrix ring.
\end{abstract}

1991 Mathematics subject classification: Primary 16S50, 16 Y30.

\section{Introduction}

Apart from structural considerations, various types of rings of matrices provide a rich source of examples and counterexamples. In fact, Rowen (see [12, p. 29]) states that rings of matrices "are undoubtedly the most widely studied class of noncommunicative rings". Generalized matrix rings form one of the largest classes of matrix rings, and in their own right these rings have been studied extensively in various contexts. See, for example, [2, 5, 6 and 7]. This class contains the class of structural matrix rings, which in turn contains the complete matrix rings and the triangular matrix rings, as well as the (complete) blocked triangular matrix rings. See, for example, $[3,4,10,14$ and 15$]$.

In [13] it was shown that the right ring associated with a finite simple abelian nearring contains a set of matrix units and is therefore isomorphic to a complete matrix ring. Secondly, a Wedderburn-Artin type result was obtained for the factor ring $\mathcal{R} / \mathcal{I}$, in the sense that $\mathcal{R} / \mathcal{I}$ is isomorphic to a direct sum of complete matrix rings, where $\mathcal{R}$ is the right ring associated with a finite abelian centralizer near-ring and $\mathcal{I}$ is a certain nilpotent ideal of $\mathcal{R}$. However, in neither of the above cases was any specific isomorphism exhibited.

Throughout the sequel $N$ will be a zero-symmetric right near-ring with identity, and we assume that $N$ is abelian, i.e. $(N,+)$ is abelian. We consider a specific subring of the ring End $(N)$ of endomorphisms of the abelian group $(N,+)$, namely the subring $\mathcal{R}$ generated by the set of right multiplication maps $\rho[n]: N \rightarrow N$, with $\rho[n](m)=m n, m, n \in N$, called the right ring associated with $N$ in [13]. Note that every element of $\mathcal{R}$ can be written as a (finite) sum $\sum_{u} \rho\left[n_{u}\right]$ of right multiplication maps, with the $n_{u}$ 's elements of $N$. 
The purpose of this paper is to describe $\mathcal{R}$ explicitly as a generalized blocked triangular matrix ring in the case where $N$ is any member of a large class of finite (abelian) centralizer near-rings. This will be done in a constructive way, i.e. an isomorphism map will be established. We will show that the mentioned generalized blocked triangular matrix ring can turn out to be a structural matrix ring.

\section{The representation of the right ring associated with a finite simple abelian near-ring as a complete matrix ring}

In this section we consider the setting in [13, Section 3]. For the ease of the reader we provide the pertinent results, definitions and notation.

A basic near-ring-theoretic result states that every finite simple zero-symmetric near-ring $N$ (with 1 ) which is not a ring is isomorphic to a centralizer near-ring

$$
M_{A}(G):=\{f: G \rightarrow G \mid f(0)=0 \text { and } f(\alpha v)=\alpha f(v), \forall \alpha \in A, \forall v \in G\},
$$

where $G$ is a finite group (written additively) and $A$ is a group of fixed point free automorphisms of $G$. Under the added assumption that $N$ be an abelian near-ring, $G$ is an abelian group.

Henceforth in this section $G$ will be a finite abelian group, $A:=\left\{\alpha_{1}, \ldots, \alpha_{s}\right\}$ will be a group of fixed point free automorphisms of $G$, and $N:=M_{A}(G)$. Let $\left\{v_{1}, \ldots, v_{t}\right\}$ be a complete set of representatives of the nonzero $A$-orbits of $G$. For $i, j=1, \ldots, t$, let $e_{i j} \in N$ be defined by $e_{i j}\left(v_{j}\right)=v_{i}$ and $e_{i j}\left(v_{k}\right)=0$ if $k \neq j$, and let $E_{i j}:=\rho\left[e_{j i}\right]$, the right multiplication map on $N$ determined by $e_{j i}$. Then $\left\{E_{i j}: 1 \leq i, j \leq t\right\}$ is a set of matrix units in $\mathcal{R}$, i.e. $E_{11}+\cdots+E_{t t}=1_{\mathcal{R}}$ and $E_{i j} E_{k l}=\delta_{j k}$ where $\delta_{j k}$ is the Kronecker delta. So much for preliminaries.

Let $\alpha \in A$, let $1 \leq i \leq t$, and define $e_{i i}^{\alpha} \in N$ by

$$
e_{i i}^{\alpha}\left(v_{i}\right)=\alpha v_{i} \quad \text { and } \quad e_{i i}^{\alpha}\left(v_{j}\right)=0 \text { if } j \neq i
$$

Then $e_{i i}^{\alpha} e_{i i}^{\beta}=e_{i i}^{\beta \alpha}, \beta \in A$. In fact, $\left(e_{11}^{\alpha}+\cdots+e_{t i}^{\alpha}\right)\left(e_{11}^{\beta}+\cdots+e_{t i}^{\beta}\right)=e_{11}^{\beta \alpha}+\cdots+e_{t t}^{\beta \alpha}$, and so

$$
\rho\left[e_{11}^{\alpha}+\cdots+e_{t t}^{\alpha}\right] \cdot \rho\left[e_{11}^{\beta}+\cdots+e_{t t}^{\beta}\right]=\rho\left[e_{11}^{\alpha \beta}+\cdots+e_{n}^{\alpha \beta}\right]
$$

Hence by $[13$, Lemma 1] the set

$$
\mathcal{T}:=\left\{a_{1} \rho\left[e_{11}^{\alpha_{1}}+\cdots+e_{t i}^{\alpha_{1}}\right]+\cdots+a_{s} \rho\left[e_{11}^{\alpha_{s}}+\cdots+e_{t i}^{\alpha_{s}}\right]: a_{i} \in \mathbb{Z}_{k}, \forall i\right\}
$$

is a subring of $\mathcal{R}$, where $k$ is the least common multiple of the orders of the elements in $G$. Let $R$ denote the subring of $\operatorname{End}(G)$ generated by $A$. Then $R$ consists of endomorphisms of $G$ of the form

$$
a_{1} \alpha_{1}+\cdots+a_{s} \alpha_{s}
$$

where $a_{i} \in \mathbb{Z}_{k}, \forall i$. 
Lemma 2.1. With the above notation, $\Phi: \mathcal{T} \rightarrow \mathcal{R}$, defined by

$$
a_{1} \rho\left[e_{11}^{\alpha_{1}}+\cdots+e_{t i}^{\alpha_{1}}\right]+\cdots+a_{s} \rho\left[e_{11}^{\alpha_{s}}+\cdots+e_{t i}^{\alpha_{s}}\right] \mapsto a_{1} \alpha_{1}+\cdots+a_{s} \alpha_{s},
$$

is a ring isomorphism.

Proof. The technique used here is based on the technique used in the proof of [13, Theorem 2]. However, we provide the details. Suppose first that

$$
\begin{aligned}
a_{1} \rho\left[e_{11}^{\alpha_{1}}+\cdots+e_{t 1}^{\alpha_{1}}\right]+\cdots+a_{s} \rho\left[e_{11}^{\alpha_{3}}+\cdots+e_{n t}^{\alpha_{3}}\right]=b_{1} \rho\left[e_{11}^{\alpha_{1}}\right. & \left.+\cdots+e_{t 1}^{\alpha_{1}}\right] \\
& +\cdots+b_{s} \rho\left[e_{11}^{\alpha_{3}}+\cdots+e_{t i}^{\alpha_{s}}\right] .
\end{aligned}
$$

Then for every $n \in N$ and every $v_{i}, i=1, \ldots, t$,

$$
a_{1} n \alpha_{1}\left(v_{i}\right)+\cdots+a_{s} n \alpha_{s}\left(v_{i}\right)=b_{1} n \alpha_{1}\left(v_{i}\right)+\cdots+b_{s} n \alpha_{s}\left(v_{i}\right)
$$

and so

$$
\left(a_{1} \alpha_{1}+\cdots+a_{s} \alpha_{s}\right) n\left(v_{i}\right)=\left(b_{1} \alpha_{1}+\cdots+b_{s} \alpha_{s}\right) n\left(v_{i}\right)
$$

Since, for a suitable choice of $n, n\left(v_{i}\right)$ can be any element of $G$, we have $a_{1} \alpha_{1}+\cdots+a_{s} \alpha_{s}=b_{1} \alpha_{1}+\cdots+b_{s} \alpha_{s}$, i.e. $\Phi$ is well defined. Furthermore, by (1) $\Phi$ is multiplicative, and it is certainly additive and onto. Lastly, suppose $a_{1} \alpha_{1}+\cdots+a_{s} \alpha_{s}=0$. Then $\left(a_{1} \alpha_{1}+\cdots+a_{s} \alpha_{s}\right) n\left(v_{i}\right)=0$ for every $n \in N$ and every $v_{i}, i=1, \ldots, t$. Hence, reversing the above steps, we have

$$
\left(a_{1} \rho\left[e_{11}^{\alpha_{1}}+\cdots+e_{t t}^{\alpha_{1}}\right]+\cdots+a_{s} \rho\left[e_{11}^{\alpha_{3}}+\cdots+e_{t t}^{\alpha_{s}}\right]\right)(n)=0
$$

for every $n \in N$, and so $a_{1} \rho\left[e_{11}^{\alpha_{1}}+\cdots+e_{t i}^{\alpha_{1}}\right]+\cdots+a_{s} \rho\left[e_{11}^{\alpha_{3}}+\cdots+e_{t i}^{\alpha_{s}}\right]=0$. Therefore $\Phi$ is $1-1$.

Let $|S|$ denote the cardinality of a set $S$. We are now in a position to exhibit an isomorphism map from $\mathcal{R}$ to $M_{t}(R)$. See [13, Theorem 2].

Theorem 2.2. Let $N:=M_{A}(G)$ be a simple abelian near-ring, where $G$ is a finite (abelian) group and $A=\left\{\alpha_{1}, \ldots, \alpha_{s}\right\}$ is a fixed point free group of automorphisms of $G$. Let $R$ be the subring of $\operatorname{End}(G)$ generated by the automorphisms in $A$. If there are $t$ nonzero $A$-orbits of $G$, then the right ring $\mathcal{R}$ associated with $N$ is isomorphic to the complete matrix ring $\mathbf{M}_{i}(R)$ via

$$
\Psi: \sum_{u} \rho\left[n_{u}\right] \mapsto\left[x_{i j}\right]
$$

with 


$$
x_{i j}:=a_{1}^{(i)} \alpha_{1}+\cdots+a_{s}^{(i)} \alpha_{s}
$$

and

$$
a_{m}^{(i)}:=\left|\left\{u: n_{u}\left(v_{i}\right)=\alpha_{m} v_{j}\right\}\right|(\bmod k), \quad m=1, \ldots, s,
$$

where $\left\{v_{1}, \ldots, v_{t}\right\}$ is a complete set of nonzero orbit representatives of $G$, and $k$ is the least common multiple of the orders of the elements in $G$.

Proof. Since $\left\{E_{i j}: 1 \leq i, j \leq t\right\}$ is a set of matrix units in $\mathcal{R}$, it follows from [9, p. 52, Proposition 6] that every element $\sum_{u} \rho\left[n_{u}\right]$ of $\mathcal{R}$ can be written in one and only one way in the form

$$
\sum_{i, j=1}^{1} b_{i j} E_{i j}
$$

where

$$
\begin{aligned}
b_{i j}:=\sum_{l=1}^{t} E_{l i}\left(\sum_{u} \rho\left[n_{u}\right]\right) E_{j l} & =\sum_{l=1}^{t} \rho\left[e_{i l}\right]\left(\sum_{u} \rho\left[n_{u}\right]\right) \rho\left[e_{l j}\right] \\
& =\sum_{l=1}^{t} \sum_{u} \rho\left[e_{l j} n_{u} e_{i l}\right] \\
& =\sum_{u} \sum_{l=1}^{t} \rho\left[e_{l j} n_{u} e_{i l}\right]
\end{aligned}
$$

Note that

$$
e_{i j} n_{u} e_{i l}= \begin{cases}e_{l l}^{\alpha_{q},} & \text { if } n_{u}\left(v_{i}\right)=\alpha_{q} v_{j}, \quad 1 \leq q \leq s \\ 0, & \text { if } n_{u}\left(v_{i}\right) \notin A v_{j}\end{cases}
$$

Hence,

$$
\sum_{l=1}^{t} \rho\left[e_{l j} n_{u} e_{i l}\right]= \begin{cases}\rho\left[e_{11}^{\alpha_{q}}+\cdots+e_{i l}^{\alpha_{q}}\right], & \text { if } n_{u}\left(v_{i}\right)=\alpha_{q} v_{j} \\ 0, & \text { if } n_{u}\left(v_{i}\right) \notin A v_{j}\end{cases}
$$

Therefore,

$$
\sum_{u} \sum_{l=1}^{i} \rho\left[e_{i j} n_{u} e_{i l}\right]=a_{1}^{(i)} \rho\left[e_{11}^{\alpha_{1}}+\cdots+e_{t t}^{a_{1}}\right]+\cdots+a_{s}^{(i j)} \rho\left[e_{11}^{\alpha_{s}}+\cdots+e_{t i}^{\alpha_{j}}\right],
$$


where $a_{m}^{(i)}=\left\{\left\{u: n_{u}\left(v_{i}\right)=\alpha_{m} v_{j}\right\} \mid(\bmod k), \quad m=1, \ldots, s\right.$. Furthermore, if $\sum_{u} \rho\left[n_{u}\right]=$ $\sum_{u^{\prime}} \rho\left[n_{u^{\prime}}^{\prime}\right]$, then the uniqueness of $b_{i j}$ in (2) ensures that

$$
\sum_{u} \sum_{l=1}^{t} \rho\left[e_{i j} n_{u} e_{i l}\right]=\sum_{u^{\prime}} \sum_{l=1}^{\ell} \rho\left[e_{i j} n_{u^{\prime}} e_{i l}\right]
$$

and so if

$$
\sum_{u^{\prime}} \sum_{l=1}^{t} \rho\left[e_{l j} n_{u^{\prime}}^{\prime} e_{i l}\right]=b_{1}^{(i j)} \rho\left[e_{11}^{\alpha_{1}}+\cdots+e_{t i}^{\alpha_{1}}\right]+\cdots+b_{s}^{(i)} \rho\left[e_{11}^{\alpha_{s}}+\cdots+e_{t t}^{\alpha_{s}}\right]
$$

with $b_{m}^{(i)}=\left|\left\{u^{\prime}: n_{u^{\prime}}^{\prime}\left(v_{i}\right)=\alpha_{m} v_{j}\right\}\right|(\bmod k), m=1, \ldots, s$, then

$$
\begin{aligned}
& a_{1}^{(i)} \rho\left[e_{11}^{\alpha_{1}}+\cdots+e_{t 1}^{\alpha_{1}}\right]+\cdots+a_{s}^{(i j)} \rho\left[e_{11}^{\alpha_{s}}+\cdots+e_{t 1}^{\alpha_{s}}\right] \\
= & b_{1}^{(i)} \rho\left[e_{11}^{\alpha_{1}}+\cdots+e_{n t}^{\alpha_{1}}\right]+\cdots+b_{s}^{(i j)} \rho\left[e_{11}^{\alpha_{s}}+\cdots+e_{t 1}^{\alpha_{s}}\right],
\end{aligned}
$$

which by Lemma 2.1 concludes the proof.

We illustrate the map in Theorem 2.2 in the following example.

Example 2.3. Let $G=\left(\mathbb{Z}_{9},+\right)$, and let $A=\{i d, \sigma\}$, where $\sigma(v):=-v, v \in \mathbb{Z}_{9}$. (See [13, Section 3, Example (b)].) Consider the elements $4 \rho\left[n_{1}\right]+\rho\left[n_{2}\right]$ and $6 \rho\left[n_{1}\right]+7 \rho\left[n_{2}\right]+$ $2 \rho\left[n_{3}\right]$ of $\mathcal{R}$, where $n_{1}, n_{2}$ and $n_{3}$ are the elements of $N\left(=M_{A}(G)\right)$ defined in the table below, and where $1,2,3$ and 4 are the representatives of the four nonzero $A$-orbits $\{1,8\},\{2,7\},\{3,6\}$ and $\{4,5\}$ respectively. Since we shall need $n_{1}^{2}, n_{2} n_{1}, n_{3} n_{1}, n_{1} n_{2}, n_{2}^{2}$ and $n_{3} n_{2}$, we include them in the table too.

\begin{tabular}{c|ccccccccc} 
& $n_{1}$ & $n_{2}$ & $n_{3}$ & $n_{1}^{2}$ & $n_{2} n_{1}$ & $n_{3} n_{1}$ & $n_{1} n_{2}$ & $n_{2}^{2}$ & $n_{3} n_{2}$ \\
\hline 1 & $\sigma(2)$ & $i d(2)$ & $i d(1)$ & $i d(2)$ & $i d(4)$ & $i d(3)$ & $\sigma(2)$ & $\sigma(4)$ & $\sigma(3)$ \\
2 & $\sigma(2)$ & $\sigma(4)$ & $\sigma(3)$ & $i d(2)$ & $i d(4)$ & $i d(3)$ & $\sigma(2)$ & $\sigma(3)$ & $i d(1)$ \\
3 & $\sigma(1)$ & $\sigma(3)$ & $i d(1)$ & $i d(2)$ & $\sigma(2)$ & $\sigma(1)$ & $i d(1)$ & $i d(3)$ & $\sigma(1)$ \\
4 & $i d(2)$ & $i d(3)$ & $\sigma(1)$ & $\sigma(2)$ & $\sigma(4)$ & $\sigma(3)$ & $\sigma(1)$ & $\sigma(3)$ & $i d(1)$
\end{tabular}

(The above notation means that, for example, $n_{1}(1)=\sigma(2)=7$.)

The function $\lambda: R \rightarrow \mathbb{Z}_{n}$, defined by

$$
a_{i} i d+a_{2} \sigma \mapsto a_{1}-a_{2}(\bmod 9),
$$

is a ring isomorphism. Let $\Lambda: \mathbf{M}_{4}(R) \rightarrow \mathbf{M}_{4}\left(\mathbb{Z}_{9}\right)$ be the induced isomorphism $\left[x_{i j}\right] \mapsto\left[\lambda\left(x_{i j}\right)\right]$. Then by Theorem 2.2 we have 


$$
\begin{aligned}
\Lambda \Psi\left(4 \rho\left[n_{1}\right]+\rho\left[n_{2}\right]\right) & =\Lambda\left(\left[\begin{array}{cccc}
0 & i+4 \sigma & 0 & 0 \\
0 & 4 \sigma & 0 & \sigma \\
4 \sigma & 0 & \sigma & 0 \\
0 & 4 i & i & 0
\end{array}\right]\right) \\
& =\left[\begin{array}{llll}
0 & 6 & 0 & 0 \\
0 & 5 & 0 & 8 \\
5 & 0 & 8 & 0 \\
0 & 4 & 1 & 0
\end{array}\right] .
\end{aligned}
$$

Similarly,

$$
\Lambda \Psi\left(6 \rho\left[n_{1}\right]+7 \rho\left[n_{2}\right]+2 \rho\left[n_{3}\right]\right)=\left[\begin{array}{llll}
2 & 1 & 0 & 0 \\
0 & 3 & 7 & 2 \\
5 & 0 & 2 & 0 \\
7 & 6 & 7 & 0
\end{array}\right]
$$

Also,

$$
\begin{aligned}
& \Lambda \Psi\left(\left(4 \rho\left[n_{1}\right]+\rho\left[n_{2}\right]\right)\left(6 \rho\left[n_{1}\right]+7 \rho\left[n_{2}\right]+2 \rho\left[n_{3}\right]\right)\right) \\
= & \Lambda \Psi\left(6 \rho\left[n_{1}^{2}\right]+\rho\left[n_{2} n_{1}\right]+8 \rho\left[n_{3} n_{1}\right]+6 \rho\left[n_{1} n_{2}\right]+7 \rho\left[n_{2}^{2}\right]+2 \rho\left[n_{3} n_{2}\right]\right) \\
= & {\left[\begin{array}{llll}
0 & 0 & 6 & 3 \\
2 & 0 & 1 & 1 \\
5 & 5 & 7 & 0 \\
5 & 3 & 3 & 8
\end{array}\right] }
\end{aligned}
$$

which equals the product of the two matrices in $\mathbb{M}_{4}\left(\mathbb{Z}_{9}\right)$ above. This is an illustration of the multiplicativity of $\Psi$ and $\Lambda \Psi$.

\section{The representation of the right ring associated with a finite abelian centralizer near-ring as a generalized blocked triangular matrix ring}

In this section $N$ will be a centralizer near-ring $M_{A}(G)$, where $\left(M_{A}(G),+\right)$ is a finite abelian group and $A$ is a group of automorphisms of the finite group $G$. We note that $G$, as pointed out in [13, Section 4], need not be abelian.

By Betsch's Lemma (see, for example, [11, Proposition 9.199]) there is a $n \in N$ such that $n(v)=w$, with $v, w \in G$, if and only if $\operatorname{stab}(v) \subseteq \operatorname{stab}(w)$, where $\operatorname{stab}(v)$ denotes the stabilizer $\{\alpha \in A: \alpha v=v\}$ of $v$, which is a subgroup of $A$. Let $\mathcal{O}_{1}, \ldots, \mathcal{O}_{t}$ denote the nonzero $A$-orbits of $G$. Consider the relations $\sim,<$ and $\lesssim$ defined on the set $\left\{\mathcal{O}_{1}, \ldots, \mathcal{O}_{t}\right\}$ as follows: 


$$
\begin{aligned}
& \mathcal{O}_{i} \sim \mathcal{O}_{j}: \Leftrightarrow \exists v_{i} \in \mathcal{O}_{i}, v_{j} \in \mathcal{O}_{j} \text { such that } \operatorname{stab}\left(v_{i}\right)=\operatorname{stab}\left(v_{j}\right) \\
& \mathcal{O}_{i}<\mathcal{O}_{j}: \Leftrightarrow \exists v_{i} \in \mathcal{O}_{i}, v_{j} \in \mathcal{O}_{j} \text { such that } \operatorname{stab}\left(v_{i}\right) \supset \operatorname{stab}\left(v_{j}\right) \\
& \mathcal{O}_{i} \lesssim \mathcal{O}_{j}: \Leftrightarrow \mathcal{O}_{i} \sim \mathcal{O}_{j} \text { or } \mathcal{O}_{i}<\mathcal{O}_{j}
\end{aligned}
$$

(The symbol $\subset$ denotes strict inclusion.)

We assume henceforth that it is possible to select the $t A$-orbit representatives $v_{1}, \ldots, v_{t}$ such that for $i, j=1, \ldots, t$,

$$
\mathcal{O}_{i} \lesssim \mathcal{O}_{j} \Leftrightarrow \operatorname{stab}\left(v_{i}\right) \supseteq \operatorname{stab}\left(v_{j}\right) \text { and } \operatorname{stab}\left(v_{i}\right) \supset \operatorname{stab}\left(v_{j}\right) \Rightarrow i>j .
$$

Therefore $\mathcal{O}_{i} \sim \mathcal{O}_{j}$ if and only if $\operatorname{stab}\left(v_{i}\right)=\operatorname{stab}\left(v_{j}\right)$. The equivalence relation $\sim$ on the $\mathcal{O}_{i}$ 's induces an equivalence relation on the $v_{i}$ 's, which we denote by $\sim$ too, in the obvious way:

$$
v_{i} \sim v_{j}: \Leftrightarrow \mathcal{O}_{i} \sim \mathcal{O}_{j}
$$

Our examples will show that condition (3), which we call the stabilizer condition, is far from being restrictive. (In fact, we have no examples of (3) not being satisfied.)

Let $p$ be the number of equivalence classes induced by the equivalence relation in (4) on the set $\left\{v_{1}, \ldots, v_{t}\right\}$, and let $v_{i_{1}}, \ldots, v_{i_{p}}$ be representatives of these $p$ equivalence classes, i.e. $v_{i_{1}}$ is an orbit representative in the first equivalence class, etc. Set $t_{m}:=\left|\left[v_{i_{m}}\right]\right|$, i.e. the cardinality of the equivalence class $\left[v_{i_{m}}\right]$ containing $v_{i_{m}}, m=1, \ldots, p$. Then $t_{1}+\cdots+t_{p}=t$, and if $\left[v_{i_{m}}\right]=\left\{v_{i_{m, 1}}, \ldots, v_{i_{m, m}}\right\}$, then by (3) and (4) we can arrange the ordering so that $\left\{i_{m, 1}, \ldots, i_{m, t_{m}}\right\}$ is a set of consecutive natural numbers, and $\left\{i_{1,1}, \ldots, i_{1, t_{1}}, \ldots, i_{p, 1}, \ldots, i_{p, l_{p}}\right\}=\{1, \ldots, t\}$.

In [13, Section 4] it was shown that the subring $\mathcal{I}$ of $\mathcal{R}$ generated by the set $\{\rho[n]$ : $n \in N$ with $\operatorname{stab}(v) \subset \operatorname{stab}(n(v)), \forall 0 \neq v \in G\}$ is a nilpotent ideal of $\mathcal{R}$, and that $\mathcal{R} / \mathcal{I}$ is isomorphic to a direct sum of complete matrix rings, with $\mathcal{R}$ the right ring associated with $N$. Consider the set $\left\{E_{11}, \ldots, E_{t u}\right\}$ of orthogonal idempotents in $\mathcal{R}$ (with sum $1_{\mathcal{R}}$ ) as in Section 2, and note that if $\operatorname{stab}\left(v_{i_{m}}\right) \nsubseteq \operatorname{stab}\left(v_{i_{l}}\right)$, with $1 \leq l, m \leq p$, then, as every element of $E_{i_{m} i_{m}} \mathcal{R} E_{i, i}$ has the form

$$
\rho\left[e_{i_{m_{m}} I_{m}}\right]\left(\sum_{u} \rho\left[n_{u}\right]\right) \rho\left[e_{i_{i} i_{i}}\right]=\sum_{u} \rho\left[e_{i_{i} i_{i}} n_{\nu} e_{i_{m} i_{m}}\right]
$$

it follows from (3) that $E_{i_{m} i_{m}} \mathcal{R} E_{i, i_{i}}=\{0\}$. Furthermore, in this case

$$
E_{i_{m, k} i_{m, k}} \mathcal{R} E_{i_{i, k} i_{l, K}}=\{0\}
$$

for all $k$ and $k^{\prime}$ such that $1 \leq k \leq t_{m}, 1 \leq k^{\prime} \leq t_{l}$, $\operatorname{since} \operatorname{stab}\left(v_{i_{m, k}}\right)=\operatorname{stab}\left(v_{i_{m}}\right) \nsubseteq \operatorname{stab}\left(v_{i}\right)=$ $\operatorname{stab}\left(v_{i_{1, k}}\right)$.

Let $y:=t_{1}+\cdots+t_{m-1}$ and $z:=t_{1}+\cdots+t_{p-1}$. We conclude from the preceding arguments and from arguments similar to those used in, for example [2, Proposition 
2.1 and Theorem 2.3], [1] or [5], that we have proved the following lemma.

Lemma 3.1. $\mathcal{R}$ is isomorphic to the $t \times t$ generalized blocked triangular matrix ring

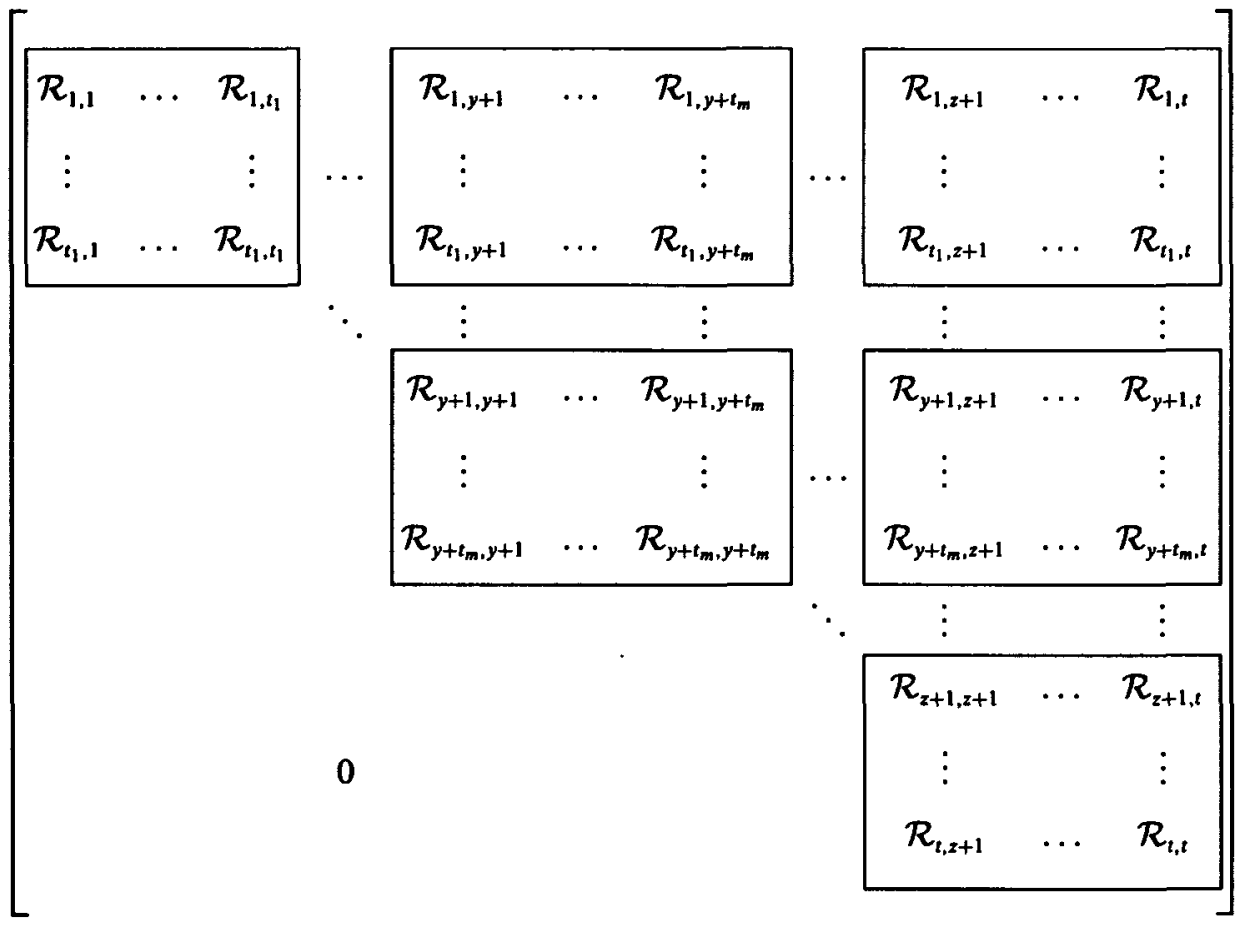

comprising all the matrices with $(i, j)$-th entries in $\mathcal{R}_{i, j}:=E_{i i} \mathcal{R} E_{j j}$ via

$$
r \mapsto E_{i j} r E_{j j}, \quad r \in \mathcal{R} .
$$

By [13, Lemma 3 and Theorem 3] every diagonal block in the generalized blocked triangular matrix ring above is isomorphic to a complete matrix ring. We now turn our attention to the $\mathcal{R}_{i j}$ 's in the blocks in the non-symmetric part of the generalized blocked triangular matrix ring in Lemma 3.1.

Let $e_{i j}: G \rightarrow G$ be defined as in Section 2. Then $e_{i j} \in N$ if and only if $\operatorname{stab}\left(v_{j}\right) \subseteq \operatorname{stab}\left(v_{i}\right)$. For such $e_{i j}$ 's we set $E_{j l}:=\rho\left[e_{i j}\right]$, as in Section 2.

For the sake of simplicity of notation, let us assume that $v_{1}, v_{2} \in\left[v_{i_{1}}\right]$ and $v_{3}, v_{4} \in\left[v_{i_{2}}\right]$, with $\operatorname{stab}\left(v_{i_{1}}\right) \subset \operatorname{stab}\left(v_{i_{2}}\right)$. Then $e_{31}, e_{41}, e_{32}, e_{42} \in N$, and so $E_{13}, E_{14}, E_{23}$, $E_{24} \in \mathcal{R}$. (Note that, for example, $e_{13} \notin N$.) Therefore $\mathcal{R}_{1,3} \cong \mathcal{R}_{2,4}$ as $\mathcal{R}_{1,1}-\mathcal{R}_{4,4^{-}}$ bimodules, and since $\mathcal{R}_{1,1} \cong \mathcal{R}_{2,2}$ and $\mathcal{R}_{3,3} \cong \mathcal{R}_{4,4}$ as rings, it follows from, for example, [14, Lemma 2.3] that $\mathcal{R}_{1,3} \cong \mathcal{R}_{2,4} \cong \mathcal{R}_{1,4} \cong \mathcal{R}_{2,3}$ as $\mathcal{R}_{11}-\mathcal{R}_{33}$-bimodules. Consequently all the $\mathcal{R}_{i, j}$ 's in a specific non-symmetric block are isomorphic as bimodules over the appropriate rings. 
However, merely stated as in Lemma 3.1 , and taking into consideration the preceding paragraph, one has not become any wiser as far as the structure of the $\mathcal{R}_{i j}$ 's in the non-symmetric blocks is concerned. After all, for example, the ring $\mathbb{Z}_{6}$ contains the idempotents 3 and 4 , and $3+4=1_{z_{6}}$, and so $\mathbb{Z}_{6}$ is isomorphic to the $2 \times 2$ generalized matrix ring $\left[\begin{array}{ll}3 \mathbb{Z}_{6} 3 & 3 \mathbb{Z}_{6} 4 \\ 4 \mathbb{Z}_{6} 3 & 4 \mathbb{Z}_{6} 4\end{array}\right]$. Therefore, only after studying the four components does one realize that this isomorphism is merely the fact that $\mathbb{Z}_{6} \cong\left[\begin{array}{cc}\mathbb{Z}_{2} & 0 \\ 0 & \mathbb{Z}_{3}\end{array}\right] \cong \mathbb{Z}_{2} \oplus \mathbb{Z}_{3}$

The purpose of this section is to find the structure of the components $\mathcal{R}_{i, j}$ in Lemma 3.1 in order to eventually provide (Theorem 3.2) an explicit representation of $\mathcal{R}$ as a $t \times t$ generalized blocked triangular matrix ring.

Henceforth we set

$$
\begin{aligned}
& K_{m}:=\operatorname{stab}\left(v_{i_{m}}\right), \\
& H_{m}:=\left\{w \in G: \operatorname{stab}\left(v_{i_{m}}\right) \subseteq \operatorname{stab}(w)\right\},
\end{aligned}
$$

$m=1, \ldots, p$. Also, let $k_{m}$ denote the least common multiple of the elements of the (abelian) subgroup $H_{m}$ of $G$, as in [13, Section 4]. For the sake of simplicity of notation let us assume that

$$
i_{m}=m, m=1,2,3,4, \quad \text { and } \quad K_{1} \subset K_{2} \subset K_{3} \subset K_{4} .
$$

We consider $E_{11} \mathcal{R} E_{22}$.

By [13, Lemma 2] we have $E_{11} \mathcal{R} E_{22}=E_{11} \mathcal{I} E_{22}$, since we have assumed that $\operatorname{stab}\left(v_{1}\right) \subset \operatorname{stab}\left(v_{2}\right)$, where $\mathcal{I}$ is the ideal of $\mathcal{R}$ as described in the discussion preceding Lemma 3.1. A typical element of $E_{11} \mathcal{I} E_{22}$ has the form

$$
\rho\left[e_{11}\right]\left(\sum_{u} \rho\left[n_{u}\right]\right) \rho\left[e_{22}\right]=\sum_{u} \rho\left[e_{22} n_{u} e_{11}\right]
$$

with each nonzero $e_{22} n_{u} e_{11} \in N$ such that

$$
e_{22} n_{u} e_{11}\left(v_{1}\right)=\alpha v_{2}
$$

where, by the definition of $\mathcal{I}$, the element $\alpha$ of $A$ is such that $\operatorname{stab}\left(v_{1}\right) \subset\left(\alpha v_{2}\right)$.

We claim, assuming that $K_{1} \subset K_{2}$ (see (6)), that for $\alpha \in A$,

$$
\left.\operatorname{stab}\left(v_{1}\right) \subset \operatorname{stab}\left(\alpha v_{2}\right) \Leftrightarrow \alpha\right|_{H_{2}} \in \operatorname{End}\left(H_{2}, H_{1}\right),
$$

where $\operatorname{End}\left(H_{2}, H_{1}\right)$ denotes the group of endomorphisms from $H_{2}$ to $H_{1}$. (Note that End $\left(H_{2}, H_{1}\right)$ is abelian, since the $H_{i}$ 's are abelian. See [13, Section 4].) To this end, 
suppose that $\operatorname{stab}\left(v_{1}\right) \subset \operatorname{stab}\left(\alpha v_{2}\right)$, and let $w \in H_{2}$. Then, by (5), (6) and, for example [11. Proposition 9.201], $\operatorname{stab}(\alpha w)=\alpha \operatorname{stab}(w) \alpha^{-1} \supseteq \alpha \operatorname{stab}\left(v_{2}\right) \alpha^{-1}=\operatorname{stab}\left(\alpha v_{2}\right) \supset \operatorname{stab}\left(v_{1}\right)$, and so $\alpha w \in H_{1}$. Conversely, if $\left.\alpha\right|_{H_{2}} \in \operatorname{End}\left(H_{2}, H_{1}\right)$, then, as $v_{2} \in H_{2}$, we have $\alpha v_{2} \in H_{1}$. Thus $\operatorname{stab}\left(v_{1}\right) \subseteq \operatorname{stab}\left(\alpha v_{2}\right)$, and since $\left|\operatorname{stab}\left(v_{1}\right)\right|<\left|\operatorname{stab}\left(v_{2}\right)\right|=\left|\alpha \operatorname{stab}\left(v_{2}\right) \alpha^{-1}\right|=\left|\operatorname{stab}\left(\alpha v_{2}\right)\right|$, it follows that $\operatorname{stab}\left(v_{1}\right) \subset \operatorname{stab}\left(\alpha v_{2}\right)$, which establishes (8).

Note that every $\alpha \in N\left(K_{2}\right)$ satisfies (8), where $N\left(K_{2}\right)$ denotes the normalizer of $K_{2}$ in $A$, since for every $\alpha \in N\left(K_{2}\right)$ we have $\operatorname{stab}\left(\alpha v_{2}\right)=\alpha \operatorname{stab}\left(v_{2}\right) \alpha^{-1}=\operatorname{stab}\left(v_{2}\right) \supset \operatorname{stab}\left(v_{1}\right)$. Moreover, arguments similar to the preceding ones show that

$$
\left.\alpha \in N\left(k_{2}\right) \Leftrightarrow \alpha\right|_{H_{2}} \in \operatorname{Aut}\left(H_{2}\right) .
$$

These results tie in with the fact that $\operatorname{Aut}\left(H_{2}\right) \subseteq \operatorname{End}\left(H_{2}, H_{1}\right)$.

Furthermore, with $e_{22} n_{4} e_{11}\left(v_{1}\right)=\alpha v_{2}$ as in (7) and (8), $\left(\rho\left[e_{22} n_{u} e_{11}\right](n)\right)\left(v_{1}\right)=\alpha n\left(v_{2}\right)$ for every $n \in M_{A}(G)$. Since stab $\left(v_{2}\right) \subseteq \operatorname{stab}\left(n\left(v_{2}\right)\right)$, it follows that $n\left(v_{2}\right) \in H_{2}$, and so the idea in the proof of [13, Lemma 1] shows that the additive order of $\rho\left[e_{22} n_{u} e_{11}\right]$ is $k_{2}$.

Next, let $A_{12}$ be the set of left cosets $\bar{\alpha}:=\alpha K_{2}$ of $K_{2}$ in $A$, with $\alpha$ as in (8), i.e.

$$
A_{12}:=\left\{\bar{\alpha}: \operatorname{stab}\left(v_{1}\right) \subset \operatorname{stab}\left(\alpha v_{2}\right)\right\} .
$$

Then $A_{2} \subseteq A_{12}$, with $A_{2}=N\left(K_{2}\right) / K_{2}$ as in [13, Section 4]. Let $\left[A: N\left(K_{2}\right)\right]$ denote the index of $N\left(K_{2}\right)$ in $A$. We note that if $\left[A: N\left(K_{2}\right)\right]=2$ and $\operatorname{stab}\left(v_{1}\right) \subset \operatorname{stab}\left(\alpha v_{2}\right)$ for some $\alpha \in A \backslash N\left(K_{2}\right)$, then $\operatorname{stab}\left(v_{1}\right) \subset \operatorname{stab}\left(\beta v_{2}\right)$ for all $\beta \in A \backslash N\left(K_{2}\right)$, since by, for example, [8, Corollary II.4.4 (iii)], the number of subgroups of $A$ conjugate to $K_{2}$ equals $\left[A: N\left(K_{2}\right)\right]$, and so if $\beta \in A \backslash N\left(K_{2}\right)$, then $\operatorname{stab}\left(\beta v_{2}\right)=\beta \operatorname{stab}\left(v_{2}\right) \beta^{-1} \neq \operatorname{stab}\left(v_{2}\right)$, which implies that $\operatorname{stab}\left(\beta v_{2}\right)=\operatorname{stab}\left(\alpha v_{2}\right) \supset \operatorname{stab}\left(v_{1}\right)$.

In general, with $K_{l} \subset K_{m}, 1 \leq l \leq m \leq p$, we set

$$
A_{m}:=\left\{\bar{\alpha}_{m, 1}, \ldots, \bar{\alpha}_{m, q_{m}}\right\} \text { and } A_{l m}:=\left\{\bar{\alpha}_{m, 1}, \ldots, \bar{\alpha}_{m, q_{m}}, \ldots, \bar{\alpha}_{m, q_{m}(n)}\right\} \text {. }
$$

We may view $A_{l m}$, in the light of (8), as a subset of $\operatorname{End}\left(H_{m}, H_{l}\right)$ by setting

$$
\bar{\alpha} w:=\alpha w,
$$

$\bar{\alpha} \in A_{w l}, w \in H_{2}$. Since $\alpha v_{m}=\beta v_{m}$ if and only if $\alpha^{-1} \beta \in \operatorname{stab}\left(v_{m}\right)$ if and only if $\alpha K_{m}=\beta K_{m}$, it follows from (7) that if $n_{m, j} \in N$ is defined by

$$
n_{m, j}\left(v_{l}\right)=\alpha_{m, j} v_{m} \text { and } n_{m, j}(v)=0 \text { if } v \notin A v_{l},
$$

$j=1, \ldots, q_{m}^{()}$, then the foregoing results show that $E_{i i q} \mathcal{R} E_{i_{m i m}}$ is the subgroup

$$
\left\{a_{1} \rho\left[n_{m, 1}\right]+\cdots+a_{q_{m}^{(m)}} \rho\left[n_{m, q_{m}^{(0)}}\right]: a_{j} \in \mathbb{Z}_{k_{m}}, \forall j\right\}
$$

of $(\operatorname{End}(N),+)$. Since $k_{m}$ is the least common multiple of the elements of $H_{m}$, every element of $\operatorname{End}\left(H_{m}, H_{l}\right)$ has additive order which divides $k_{m}$. Therefore the subgroup of 
$\operatorname{End}\left(H_{m}, H_{l}\right)$ generated by $A_{l m}$ is

$$
S_{l m}:=\left\{a_{1} \bar{\alpha}_{m, 1}+\cdots+a_{q_{m}} \bar{\alpha}_{m, q_{m}^{(0)}}: a_{j} \in \mathbb{Z}_{k_{m}}, \forall j\right\}
$$

Then we claim that

$$
E_{i, i} \mathcal{R} E_{i_{m} i_{m}} \cong S_{l m}
$$

as groups via

$$
\Phi_{l m}: a_{1} \rho\left[n_{m, 1}\right]+\cdots+a_{q_{m}^{(n)}} \rho\left[n_{m, q_{m}^{(0)}}\right] \mapsto a_{1} \bar{\alpha}_{m, 1}+\cdots+a_{q_{m}^{(n)}} \bar{\alpha}_{m, q_{m}^{(0)}}
$$

We now verify (13). For the sake of simplicity of notation, and without loss of generality, we set $l=1$ and $m=2$, and we use (6). Suppose first that $a_{1} \rho\left[n_{2,1}\right]+$ $\cdots+a_{q_{2}^{(1)}} \rho\left[n_{2, q_{2}^{(1)}}\right]=b_{1} \rho\left[n_{2,1}\right]+\cdots+b_{q_{2}^{(1)}} \rho\left[n_{2, q_{2}^{(1)}}\right]$. Then, as in the proof of $[13$, Theorem 2] it follows from (11) that, for every $n_{2} \in N$,

$$
\begin{aligned}
\left(a_{1} \alpha_{2,1}+\cdots+a_{q_{2}^{(1)}} \alpha_{2, q_{2}^{(1)}}\right) n\left(v_{2}\right) & =\left(\left(a_{1} \rho\left[n_{2,1}\right]+\cdots+a_{q_{2}^{(1)}} \rho\left[n_{2, q_{2}^{(1)}}\right]\right)(n)\right)\left(v_{1}\right) \\
& =\left(\left(b_{1} \rho\left[n_{2,1}\right]+\cdots+b_{q_{2}^{(1)}} \rho\left[n_{2, q_{2}^{(1)}}\right]\right)(n)\right)\left(v_{1}\right) \\
& =\left(b_{1} \alpha_{2,1}+\cdots+b_{q_{2}^{(1)}} \alpha_{2, q_{2}^{(1)}}\right) n\left(v_{2}\right) .
\end{aligned}
$$

Now $n\left(v_{2}\right)$ is an arbitrary element $w$ of $H_{2}$, and so by (10)

$$
a_{1} \bar{\alpha}_{2,1}+\cdots+a_{q_{2}^{(1)}} \bar{\alpha}_{2, q_{2}^{(1)}}=b_{1} \bar{\alpha}_{2,1}+\cdots+b_{q_{2}^{(1)}} \bar{\alpha}_{2, q_{2}^{(1)}}
$$

i.e. $\Phi_{12}$ is well defined. By (12) $\Phi$ is onto, and it is certainly additive. Lastly, if $a_{1} \bar{\alpha}_{2,1}+\cdots+a_{q_{2}^{(1)}} \bar{\alpha}_{2, q_{2}^{(1)}}=0$, then $\left(a_{1} \bar{\alpha}_{2,1}+\cdots+a_{q_{2}^{(1)}} \bar{\alpha}_{2, q_{2}^{(1)}}\right) n\left(v_{2}\right)=0$ for every $n \in N$, and reversing the above steps it follows that

$$
\left(\left(a_{1} \rho\left[n_{2,1}\right]+\cdots+a_{q_{2}^{(1)}} \rho\left[n_{2, q_{2}^{(1)}}\right]\right)(n)\right)\left(v_{1}\right)=0 .
$$

The second part of (11) now shows that $a_{1} \rho\left[n_{2,1}\right]+\cdots+a_{q_{2}^{(1)}} \rho\left[n_{2, q_{2}^{(1)}}\right]=0$, which establishes (13).

For $m=1, \ldots, p$, set

$$
R_{m}:=\left\{a_{1} \bar{\alpha}_{m, 1}+\cdots+a_{q_{m}} \bar{\alpha}_{m, q_{m}}: a_{j} \in \mathbb{Z}_{k_{m}}, \forall j\right\}
$$

By (9) and (12) $R_{m} \subseteq S_{l m}$ as sets, and $R_{m}$ is the subring of Aut $\left(H_{m}\right)$ generated by the elements of $A_{m}$ (see [13, Section 4]). Note also that if $K_{l}, \subset K_{l} \subset K_{m}$, with $1 \leq l^{\prime}<l<m \leq p$, then, as sets, $S_{l m} \subseteq S_{l^{\prime} m}$. Moreover, if $K_{m}$ is a normal subgroup of $A$, then, as sets, 


$$
R_{m}=S_{l m}=S_{l^{\prime} m}
$$

We now turn $S_{l m}$ into an $R_{l}-R_{m}$-bimodule. From [13, Section 4] we know that

$$
E_{i_{m} i_{m}} \mathcal{R} E_{i_{m} i_{m}}=\left\{b_{1} \rho\left[n_{m, 1}\right]+\cdots+b_{q_{m}} \rho\left[n_{m, q_{m}}\right]: b_{j} \in \mathbb{Z}_{k_{m}}, \forall j\right\}
$$

and

$$
\Phi_{m}: E_{i_{m} i_{m}} \mathcal{R} E_{i_{m} i_{m}} \rightarrow R_{m}
$$

defined by

$$
b_{1} \rho\left[n_{m, 1}\right]+\cdots+b_{q_{m}} \rho\left[n_{m, q_{m}}\right] \mapsto b_{1} \bar{\alpha}_{m, 1}+\cdots+b_{q_{m}} \bar{\alpha}_{m, q_{m}},
$$

is a ring isomorphism. Together with the group isomorphism $\Phi_{l m}: E_{i i_{i} i} \mathcal{R} E_{i_{m} i_{m}} \rightarrow S_{l m}$ in (13) we obtain $S_{l m}$ naturally as a right $R_{m}$-module via

$$
s_{l m} \cdot r_{m}=\Phi_{l m}\left(\Phi_{l m}^{-1}\left(s_{l m}\right) \Phi_{m}^{-1}\left(r_{m}\right)\right)
$$

$s_{l m} \in S_{l m}, r_{m} \in R_{m}$. Similarly, we obtain $S_{l m}$ as a left $R_{l}$-module; in fact, $S_{l m}$ is an $R_{l}-R_{m}-$ bimodule.

In the light of Lemma 3.1 it should now be clear, again keeping (6) in mind, that

$$
\phi_{13}^{(2)}: S_{12} \otimes_{R_{2}} S_{23} \rightarrow S_{13}, \quad \text { defined by }\left(s_{12}, s_{23}\right) \mapsto \Phi_{13}\left(\Phi_{12}^{-1}\left(s_{12}\right) \Phi_{23}^{-1}\left(s_{23}\right)\right) \text {, }
$$

$s_{12} \in S_{12}, s_{23} \in S_{23}$, is an $R_{1}-R_{3}$-homomorphism, and that, with $\varphi_{14}^{(2)}, \varphi_{14}^{(3)}$ and $\varphi_{24}^{(3)}$ defined similarly, the diagram

$$
\begin{aligned}
& S_{12} \otimes_{R_{2}} S_{23} \otimes_{R_{3}} S_{34} \stackrel{i d_{12} \otimes \varphi_{24}^{(3)}}{\longrightarrow} S_{12} \otimes_{R_{2}} S_{24} \\
& \downarrow \varphi_{13}^{(2)} \otimes i d_{34} \quad \downarrow \varphi_{14}^{(2)} \\
& S_{13} \otimes_{R_{3}} S_{34} \quad \stackrel{\varphi_{14}^{(3)}}{\longrightarrow} \quad S_{14}
\end{aligned}
$$

commutes, where $i d_{l m}$ denotes the identity function on $S_{l m}$.

For $1 \leq l<m \leq t$, we set

$$
T_{l m}:= \begin{cases}S_{l m}, & \text { if } K_{1} \subset K_{m} \\ \{0\}, & \text { otherwise; }\end{cases}
$$

Thus, armed with the foregoing results, we conclude from Theorem 2.2, Lemma 3.1 and, for example, [1] or [7], that we have proved our main result: 
Theorem 3.2. Let $N$ be a centralizer near-ring $M_{A}(G)$, where $\left(M_{A}(G),+\right)$ is a finite abelian group and $A$ is a group of automorphisms of the finite group $G$ such that the stabilizer condition is satisfied. Then, with the above notation, the right ring $\mathcal{R}$ associated with $N$ is isomorphic to the $t \times t$ generalized blocked triangular matrix ring

$$
\left[\begin{array}{ccccccc}
\mathbf{M}_{t_{1}}\left(R_{1}\right) & \ldots & \mathbf{M}_{t_{1} \times t_{1}}\left(T_{1 l}\right) & \ldots & \mathbf{M}_{t_{1} \times t_{m}}\left(T_{1 m}\right) & \ldots & \mathbf{M}_{t_{1} \times t_{p}}\left(T_{1 p}\right) \\
& \ddots & \vdots & \ddots & \vdots & \ddots & \vdots \\
& & \mathbf{M}_{t_{l}}\left(R_{l}\right) & \ldots & \mathbf{M}_{t_{1} \times t_{m}}\left(T_{t m}\right) & \ldots & \mathbf{M}_{t_{1} \times t_{p}}\left(T_{l p}\right) \\
& & \ddots & \vdots & \ddots & \vdots \\
& & & & \mathbf{M}_{t_{m}}\left(R_{m}\right) & \ldots & \mathbf{M}_{t_{m} \times t_{p}}\left(T_{m p}\right) \\
& & & & & \ddots & \vdots \\
& & & & & & \mathbf{M}_{t_{p}}\left(R_{p}\right)
\end{array}\right]
$$

via

$$
\Psi: \sum_{u} \rho\left[n_{u}\right] \mapsto\left[x_{i j}\right]
$$

where

$$
x_{i j}:=a_{1}^{(i)} \bar{\alpha}_{m, 1}+\cdots+a_{q_{m}^{(i)}}^{(i)} \bar{\alpha}_{m, q_{m}^{(i)}}
$$

with

$$
\begin{aligned}
& a_{1}^{(i)}:=\mid\left\{u: n_{u}\left(v_{i}\right)=\alpha_{m, 1} v_{j}\right\} \text { vert }\left(\bmod k_{m}\right), \ldots, a_{q_{m}^{(i)}}^{(i)}:=\left|\left\{u: n_{u}\left(v_{i}\right)=\alpha_{m, q_{m}^{(n)}} v_{j}\right\}\right|\left(\bmod k_{m}\right), \\
& \text { if } i \in\left\{i_{l, 1}, \ldots, i_{l, l_{1}}\right\}, j \in\left\{i_{m, 1}, \ldots, i_{m, l_{m}}\right\}, 1 \leq l \leq m \leq p, \text { and } q_{m}^{(m)}:=q_{m} .
\end{aligned}
$$

By [14, Section 2] the class of structural matrix rings may be viewed as a subclass of the class of generalized blocked triangular matrix rings in the sense that only one base ring plays a role in the description of any particular structural matrix ring and that no substructures of that base ring play a role in the mentioned description. To be more precise, if $B:=\left[b_{i j}\right]$ is a reflexive and transitive $n \times n$ Boolean matrix, i.e. $b_{u}=1$ for every $i$, and $b_{i j}=1=b_{j k}$ implies $b_{i k}=1,1 \leq i, j, k \leq n$, and if $R$ is any ring, then the structural matrix ring $\mathbf{M}(B, R)$ is the subring of the complete matrix ring $\mathbf{M}_{n}(R)$ comprising all matrices with 0 in position $(k, l)$ whenever $b_{k l}=0$. Recent papers on structural matrix rings, studied in their own right, include [3], [4], [15] and [16].

From Theorem 3.2 we conclude that if the rings $R_{m}, m=1, \ldots, p$, are isomorphic to one another, and if $R_{m}=S_{l m}$ (as sets) in the case $K_{l} \subset K_{m}, 1 \leq l<m \leq p$, which 
occurs if, for example, $K_{m}$ is a normal subgroup of $A$, then $\mathcal{R}$ is isomorphic to a $t \times t$ structural matrix ring over $R_{1}$. See Example 3.4.

Example 3.3. Let $G=\mathbb{Z}_{8} \oplus \mathbb{Z}_{8}$, and let $A$ be the group (under matrix multiplication) of real matrices generated by

$$
\alpha:=\left[\begin{array}{cc}
0 & 1 \\
-1 & 0
\end{array}\right] \text { and } \beta:=\left[\begin{array}{ll}
0 & 1 \\
1 & 0
\end{array}\right]
$$

acting on $G$ via matrix multiplication, where we view the elements of $\mathbb{Z}_{8} \oplus \mathbb{Z}_{8}$ as column matrices. Then (see, for example $\left[8\right.$, p. 33, Exercise 4]) $A \cong \mathcal{D}_{4}$, the dihedral group of order 8 , generated by $\alpha$ and $\beta$, with $\alpha^{4}=i d$ (the identity matrix), $\beta^{2}=i d$ and $(\alpha \beta)^{2}=i d$. Since $\alpha^{i} \beta=\beta \alpha^{4-i}$ for $0 \leq i \leq 3$, the elements of $A$ can be represented as $i d, \alpha$, $\alpha^{2}, \alpha^{3}, \beta, \alpha \beta, \alpha^{2} \beta$ and $\alpha^{3} \beta$, with

$$
\begin{aligned}
& \alpha^{2}=\left[\begin{array}{cc}
-1 & 0 \\
0 & -1
\end{array}\right], \alpha^{3}=\left[\begin{array}{cc}
0 & -1 \\
1 & 0
\end{array}\right], \\
& \alpha \beta=\left[\begin{array}{cc}
1 & 0 \\
0 & -1
\end{array}\right], \alpha^{2} \beta=\left[\begin{array}{cc}
0 & -1 \\
-1 & 0
\end{array}\right], \alpha^{3} \beta=\left[\begin{array}{cc}
-1 & 0 \\
0 & 1
\end{array}\right] .
\end{aligned}
$$

Here $t=14$, and we select the $A$-orbit representatives $v_{1}, \ldots, v_{14}$ of $\mathcal{O}_{1}, \ldots, \mathcal{O}_{14}$ as follows:

$$
\begin{aligned}
& v_{1}=\left[\begin{array}{l}
1 \\
2
\end{array}\right], v_{2}=\left[\begin{array}{l}
1 \\
3
\end{array}\right], v_{3}=\left[\begin{array}{l}
2 \\
3
\end{array}\right], v_{4}=\left[\begin{array}{l}
1 \\
0
\end{array}\right], v_{5}=\left[\begin{array}{l}
1 \\
4
\end{array}\right], v_{6}=\left[\begin{array}{l}
2 \\
0
\end{array}\right], v_{7}=\left[\begin{array}{l}
2 \\
4
\end{array}\right], \\
& v_{8}=\left[\begin{array}{l}
3 \\
0
\end{array}\right], v_{9}=\left[\begin{array}{l}
3 \\
4
\end{array}\right], v_{10}=\left[\begin{array}{l}
1 \\
7
\end{array}\right], v_{11}=\left[\begin{array}{l}
2 \\
6
\end{array}\right], v_{12}=\left[\begin{array}{l}
3 \\
5
\end{array}\right], v_{13}=\left[\begin{array}{l}
4 \\
0
\end{array}\right], v_{14}=\left[\begin{array}{l}
4 \\
4
\end{array}\right],
\end{aligned}
$$

Then

$$
\begin{gathered}
\mathcal{O}_{14}<\mathcal{O}_{13}<\mathcal{O}_{9} \sim \mathcal{O}_{8} \sim \mathcal{O}_{7} \sim \mathcal{O}_{6} \sim \mathcal{O}_{5} \sim \mathcal{O}_{4}<\mathcal{O}_{3} \sim \mathcal{O}_{2} \sim \mathcal{O}_{1} \\
\mathcal{O}_{14}<\mathcal{O}_{12} \sim \mathcal{O}_{11} \sim \mathcal{O}_{10}<\mathcal{O}_{3} \sim \mathcal{O}_{2} \sim \mathcal{O}_{1}
\end{gathered}
$$

and it can be verified that (3) is satisfied. Thus $p=5$, and the five equivalence classes induced by $\sim$ on $\left\{v_{1}, \ldots, v_{14}\right\}$ are $\left[v_{i_{1}}\right]:=\left\{v_{1}, v_{2}, v_{3}\right\},\left[v_{i_{2}}\right]:=\left\{v_{4}, v_{5}, v_{6}, v_{7}, v_{8}, v_{9}\right\}$, $v_{i 3}:=\left\{v_{10}, v_{11}, v_{12}\right\}, v_{i_{4}}:=\left\{v_{13}\right\}$ and $v_{i s}:=\left\{v_{14}\right\}$. The stabilizers $K_{m}, m=1, \ldots, 5$, are

$$
K_{1}=\{i d\}, K_{2}=\{i d, \alpha \beta\}, K_{3}=\left\{i d, \alpha^{2} \beta\right\}, K_{4}=\left\{i, \alpha^{2}, \alpha \beta, \alpha^{3} \beta\right\}, k_{5}=A,
$$

with 


$$
K_{1} \subset K_{2} \subset K_{4} \subset K_{5} \text { and } K_{1} \subset K_{3} \subset K_{5}
$$

Note that $K_{4}$ is the only non-trivial normal subgroup of $A$ amongst the $K_{m}$ 's. By Theorem $3.2 \mathcal{R}$ is isomorphic to the $14 \times 14$ generalized blocked triangular matrix ring

$$
\left[\begin{array}{ccccc}
\mathbf{M}_{3}\left(R_{1}\right) & \mathbf{M}_{3 \times 6}\left(S_{12}\right) & \mathbf{M}_{3 \times 3}\left(S_{13}\right) & \mathbf{M}_{3 \times 1}\left(R_{4}\right) & \mathbf{M}_{3 \times 1}\left(R_{5}\right) \\
& \mathbf{M}_{6}\left(R_{2}\right) & 0 & \mathbf{M}_{6 \times 1}\left(R_{4}\right) & \mathbf{M}_{6 \times 1}\left(R_{5}\right) \\
& \mathbb{M}_{3}\left(R_{3}\right) & 0 & \mathbb{M}_{3 \times 1}\left(R_{5}\right) \\
& 0 & & R_{4} & R_{5} \\
& & & & R_{5}
\end{array}\right]
$$

where we use the set equality in (15), and where the zeros denote the zero matrices of the appropriate sizes.

Direct calculations show that $k_{1}=k_{2}=k_{3}=8$ and $k_{4}=k_{5}=2$. Since $\alpha^{2}=-i d$, $\alpha^{3} \beta=-\alpha \beta, \alpha^{2} \beta=-\beta$ and $\alpha^{3}=-\alpha$, it follows readily that the ring $R_{1}$ is isomorphic to the subring

$$
\left\{\left[\begin{array}{ll}
a & c \\
d & b
\end{array}\right]: a, b, c, d \in \mathbb{Z}_{8}, a-b, c-d \in \mathbb{Z}_{8}\right\}
$$

of $\mathbf{M}_{2}\left(\mathbb{Z}_{8}\right)$ via

$$
\begin{gathered}
\left\{a_{1} i d+a_{2} \alpha+a_{3} \alpha^{2}+a_{4} \alpha^{3}+a_{5} \beta+a_{6} \alpha \beta+a_{7} \alpha^{2} \beta+a_{8} \alpha^{3} \beta: a_{i} \in \mathbb{Z}_{8}, \forall i\right\} \\
\mapsto\left[\begin{array}{ll}
\left(a_{1}-a_{3}\right)+\left(a_{6}-a_{8}\right) & \left(a_{5}-a_{7}\right)+\left(a_{2}-a_{4}\right) \\
\left(a_{5}-a_{7}\right)-\left(a_{2}-a_{4}\right) & \left(a_{1}-a_{3}\right)-\left(a_{6}-a_{8}\right)
\end{array}\right] .
\end{gathered}
$$

Next, $N\left(K_{2}\right) / K_{2}=\left\{i d K_{2}, \alpha^{2} K_{2}\right\}$, and since $\alpha^{2}=-i d$, it follows that $R_{2} \cong \mathbb{Z}_{8}$ as rings. Similarly, $R_{3} \cong \mathbb{Z}_{8}$ and $R_{5} \cong \mathbb{Z}_{2}$ as rings. However, $N\left(K_{4}\right) / K_{4}=\left\{i d K_{4}, \alpha K_{4}\right\}$ and so $R_{4} \cong \mathbb{Z}_{2}\left[\mathbb{Z}_{2}\right]$, the group ring of (the additive group) $\mathbb{Z}_{2}$ over (the field) $\mathbb{Z}_{2}$. Similarly, $S_{12} \cong S_{13} \cong\left(\mathbb{Z}_{8}\left[\mathbb{Z}_{2}\right],+\right)$, and so

$$
\mathcal{R} \cong\left[\begin{array}{ccccc}
\mathbf{M}_{3}\left(R_{1}\right) & \mathbf{M}_{3 \times 6}\left(\mathbb{Z}_{8}\left[\mathbb{Z}_{2}\right]\right) & \mathbf{M}_{3 \times 3}\left(\mathbb{Z}_{8}\left[\mathbb{Z}_{2}\right]\right) & \mathbf{M}_{3 \times 1}\left(\mathbb{Z}_{2}\left[\mathbb{Z}_{2}\right]\right) & \mathbf{M}_{3 \times 1}\left(\mathbb{Z}_{2}\right) \\
& \mathbf{M}_{6}\left(\mathbb{Z}_{8}\right) & 0 & \mathbf{M}_{6 \times 1}\left(\mathbb{Z}_{2}\left[\mathbb{Z}_{2}\right]\right) & \mathbf{M}_{6 \times 1}\left(\mathbb{Z}_{2}\right) \\
& & \mathbf{M}_{3}\left(\mathbb{Z}_{8}\right) & 0 & \mathbf{M}_{3 \times 1}\left(\mathbb{Z}_{2}\right) \\
& 0 & & \mathbb{Z}_{2}\left[\mathbb{Z}_{2}\right] & \mathbb{Z}_{2} \\
& & & & \mathbb{Z}_{2}
\end{array}\right]
$$


Since we have already provided an illustration of $\Psi$ (see Example 2.3) as far as the diagonal blocks, i.e. the complete matrix rings $\mathbb{M}_{t_{m}}\left(R_{m}\right)$, are concerned, we conclude this example by illustrating the multiplicativity of $\Psi^{m}$ as regards the non-symmetric part of the above generalized blocked triangular matrix ring by using (17) and (18).

Let $n_{u} \in N, u=1, \ldots, 15$, be defined as in the following table:

\begin{tabular}{|c|c|c|c|c|c|c|c|c|c|c|c|c|c|c|c|}
\hline & $n_{1}$ & $n_{2}$ & $n_{3}$ & $n_{4}$ & $n_{5}$ & $n_{6}$ & $n_{7}$ & $n_{8}$ & $n_{9}$ & $n_{10}$ & $n_{11}$ & $n_{12}$ & $n_{13}$ & $n_{14}$ & $n_{15}$ \\
\hline $\begin{array}{l}v_{1} \\
v_{4}\end{array}$ & $i d v_{1}$ & $\alpha v_{1}$ & $\alpha^{2} v_{1}$ & $\alpha \beta v_{4}$ & $\alpha^{2} \beta v_{4}$ & $\beta v_{13}$ & $\alpha \beta v_{13}$ & $\alpha^{2} \beta v_{14}$ & $\begin{array}{c}\alpha^{3} \beta v_{4} \\
\alpha \beta v_{4}\end{array}$ & $\alpha^{3} \beta v_{4}$ & $\alpha^{3} v_{4}$ & $\alpha^{2} v_{4}$ & $\begin{array}{c}\alpha^{3} v_{13} \\
\alpha^{2} \beta v_{13}\end{array}$ & $\beta v_{14}$ & $\alpha^{3} \beta v_{14}$ \\
\hline $\begin{array}{l}v_{13} \\
v_{14}\end{array}$ & & & & & & & & & & & & & & $\alpha v_{14}$ & $\alpha \beta v_{14}$ \\
\hline
\end{tabular}

(The table is read as follows: $n_{9}\left(v_{1}\right)=\alpha^{3} \beta v_{4}, n_{9}\left(v_{4}\right)=\alpha \beta v_{4}$, and $n_{9}$ is 0 on the other twelve nonzero $A$-orbits.)

Since $A_{12}=\left\{i d K_{2}, \alpha K_{2}, \alpha^{2} K_{2}, \alpha^{3} K_{2}\right\}, A_{13}=\left\{i d K_{3}, \alpha K_{3}, \alpha^{2} K_{3}, \alpha_{3} K_{3}\right\}$ and $A_{4}=A_{14}=$ $A_{24}=\left\{i d K_{4}, \alpha K_{4}\right\}$, and since $\alpha^{2}=-i d$ and $\alpha^{3}=-\alpha$, we use the following representation for $S_{12}, S_{13}$ and $R_{4}=S_{14}=S_{24}$ :

$$
\begin{aligned}
& S_{12}=\left\{a_{1} \overline{i d}+a_{2} \bar{\alpha}: a_{1}, a_{2} \in \mathbb{Z}_{8}\right\} \text { (where the cosets are the left cosets of } K_{2} \text { in } A \text { ), } \\
& S_{13}=\left\{b_{1} \overline{i d}+b_{2} \bar{\alpha}: b_{1}, b_{2} \in \mathbb{Z}_{8}\right\} \text { (left cosets of } K_{3} \text { in } A \text { ), } \\
& R_{4}=S_{14}=S_{24}=\left\{c_{1} \overline{i d}+c_{2} \bar{\alpha}: c_{1}, c_{2} \in \mathbb{Z}_{2}\right\} \text { (left cosets of } K_{4} \text { in } A \text { ). }
\end{aligned}
$$

The other representations should now be clear.

Let $V, W \in \mathcal{R}$ be the following:

$$
\begin{aligned}
& V:=\sum_{i=1}^{8} a_{i} \rho\left[n_{i}\right]=4 \rho\left[n_{1}\right]+5 \rho\left[n_{2}\right]+\rho\left[n_{3}\right]+\rho\left[n_{4}\right]+3 \rho\left[n_{5}\right]+\rho\left[n_{6}\right]+7 \rho\left[n_{7}\right]+5 \rho\left[n_{8}\right] \\
& W:=\sum_{j=9}^{15} b_{j} \rho\left[n_{j}\right]=2 \rho\left[n_{9}\right]+\rho\left[n_{10}\right]+\rho\left[n_{11}\right]+5 \rho\left[n_{12}\right]+3 \rho\left[n_{13}\right]+7 \rho\left[n_{14}\right]+5 \rho\left[n_{15}\right]
\end{aligned}
$$

(Here $a_{1}=4, \ldots, a_{8}=5$, and $b_{9}=2, \ldots, b_{15}=5$.) Then by Theorem 3.2 the first row of the $14 \times 14$ matrix $\Psi(V)$ is

$$
\left[\begin{array}{llllllllllllll}
3 i d+5 \alpha & 0 & 0 & i d K_{2}+3 \alpha K_{2} & 0 & 0 & 0 & 0 & 0 & 0 & 0 & 0 & i d K_{4}+\alpha K_{4} & 1
\end{array}\right] .
$$

We abbreviate this representation for $\Psi(V)$ by

$$
(3 i d+5 \alpha) \epsilon_{1,1}+\left(i d K_{2}+3 \alpha K_{2}\right) \epsilon_{1,4}+\left(i d K_{4}+\alpha K_{4}\right) \epsilon_{1,13}+\epsilon_{1,14}
$$

using $\epsilon_{i, j}$ to denote the matrix with 1 in position $(i, j)$ and zeros elsewhere. Similarly, 


$$
\begin{aligned}
\Psi(W)= & \left(6 i d K_{2}+7 \alpha K_{2}\right) \epsilon_{1,4}+\left(\alpha K_{4}\right) \epsilon_{1,13}+\epsilon_{1,14}+\left(3 i d K_{2}\right) \epsilon_{4,4}+\left(\alpha K_{4}\right) \epsilon_{4,13} \\
& +\epsilon_{4,14}+\epsilon_{13,14}+\epsilon_{14,14} .
\end{aligned}
$$

Now $V W=\sum_{i=1}^{8} \sum_{j=9}^{15} a_{i} b_{j} \rho\left[n_{j} n_{\mathrm{i}}\right]$, and

$$
\begin{aligned}
\left(\sum_{i=1}^{8} \sum_{j=9}^{15} a_{i} b_{j} n_{j} n_{i}\right)\left(v_{1}\right)= & \left(7 i d+7 \alpha+\alpha^{2}+7 \alpha^{2}+28 \beta+2 \alpha \beta+14 \alpha^{3} \beta\right) v_{4} \\
& +\left(24 i d+3 \alpha+15 \alpha^{3}\right) v_{13} \\
& +\left(25 \alpha+5 \alpha+15 \alpha^{3}+77 \beta+35 \alpha \beta+7 \alpha^{2} \beta+7 \alpha^{3} \beta\right) v_{14},
\end{aligned}
$$

and $\sum_{i=1}^{8} \sum_{j=9}^{15} a_{i} b_{j} n_{j} n_{i}$ is 0 on the other thirteen orbits. Hence, again by Theorem 3.2,

$$
\Psi(V W)=\left(2 i d K_{2}+4 \alpha K_{2}\right) \epsilon_{1,4}+\epsilon_{1,14},
$$

which equals the product of the matrices $\Psi(V)$ and $\Psi(W)$.

In the following example the generalized blocked triangular matrix ring in Theorem 3.2 turns out to be a structural matrix ring. (See the discussion following Theorem 3.2.)

Example 3.4. Let $A=\{i d, \alpha, \beta, \epsilon, \gamma, \delta\}$, where the elements of $A$ are the following elements of $\mathbf{M}_{4}\left(\mathbb{Z}_{2}\right)$ : id is the $4 \times 4$ identity matrix and

$$
\begin{aligned}
& \alpha:=\left[\begin{array}{llll}
1 & 0 & 0 & 1 \\
0 & 1 & 0 & 0 \\
0 & 0 & 1 & 0 \\
0 & 0 & 0 & 1
\end{array}\right], \beta:=\left[\begin{array}{llll}
1 & 0 & 0 & 0 \\
0 & 1 & 0 & 0 \\
0 & 0 & 1 & 0 \\
1 & 0 & 0 & 1
\end{array}\right]\left(=\alpha^{T}\right), \\
& \epsilon:=\left[\begin{array}{llll}
0 & 0 & 0 & 1 \\
0 & 1 & 0 & 0 \\
0 & 0 & 1 & 0 \\
1 & 0 & 0 & 0
\end{array}\right], \gamma:=\left[\begin{array}{llll}
0 & 0 & 0 & 1 \\
0 & 1 & 0 & 0 \\
0 & 0 & 1 & 0 \\
1 & 0 & 0 & 1
\end{array}\right], \delta:=\left[\begin{array}{llll}
1 & 0 & 0 & 1 \\
0 & 1 & 0 & 0 \\
0 & 0 & 1 & 0 \\
1 & 0 & 0 & 0
\end{array}\right] .
\end{aligned}
$$

Under matrix multiplication $A$ is a group which is isomorphic to the symmetric group $\mathcal{S}_{3}$; in fact, the multiplication table for $A$ is the following:

\begin{tabular}{c|ccccc} 
& $\alpha$ & $\beta$ & $\epsilon$ & $\gamma$ & $\delta$ \\
\hline$\alpha$ & $i d$ & $\gamma$ & $\delta$ & $\beta$ & $\epsilon$ \\
$\beta$ & $\delta$ & $i d$ & $\gamma$ & $\epsilon$ & $\alpha$ \\
$\epsilon$ & $\gamma$ & $\delta$ & $i d$ & $\alpha$ & $\beta$ \\
$\gamma$ & $\epsilon$ & $\alpha$ & $\beta$ & $\delta$ & $i d$ \\
$\delta$ & $\beta$ & $\epsilon$ & $\alpha$ & $i d$ & $\gamma$
\end{tabular}


So under matrix multiplication $A$ may be viewed as a group of automorphisms of $G:=\mathbb{Z}_{2} \oplus \mathbb{Z}_{2} \oplus \mathbb{Z}_{2} \oplus \mathbb{Z}_{2}$. In this case there are seven nonzero $A$-orbits of $G$. Let the orbit representatives be as follows:

$$
v_{1}=\left[\begin{array}{l}
1 \\
0 \\
0 \\
0
\end{array}\right], v_{2}=\left[\begin{array}{l}
1 \\
1 \\
0 \\
0
\end{array}\right], v_{3}=\left[\begin{array}{l}
1 \\
0 \\
1 \\
0
\end{array}\right], v_{4}=\left[\begin{array}{l}
1 \\
1 \\
1 \\
0
\end{array}\right], v_{5}=\left[\begin{array}{l}
0 \\
1 \\
0 \\
0
\end{array}\right], v_{6}=\left[\begin{array}{l}
0 \\
0 \\
1 \\
0
\end{array}\right], v_{7}=\left[\begin{array}{l}
0 \\
1 \\
1 \\
0
\end{array}\right] .
$$

Then $\mathcal{O}_{7} \sim \mathcal{O}_{6} \sim \mathcal{O}_{5}<\mathcal{O}_{4} \sim \mathcal{O}_{3} \sim \mathcal{O}_{2} \sim \mathcal{O}_{1}$, and (3) is satisfied. Furthermore, $K_{1}=\{i d, \alpha\} \subset K_{2}=A, N\left(K_{1}\right)=K_{1}$ and $k_{1}=2=k_{2}$. Therefore $\mathcal{R}$ is isomorphic to the following $7 \times 7$ structural matrix ring over $\mathbb{Z}_{2}$ :

$$
\left[\begin{array}{cc}
\mathbb{M}_{4}\left(\mathbb{Z}_{2}\right) & \mathbb{M}_{4 \times 3}\left(\mathbb{Z}_{2}\right) \\
0 & \mathbb{M}_{3}\left(\mathbb{Z}_{2}\right)
\end{array}\right]
$$

Acknowledgement. This paper was written while the second author was visiting the Department of Mathematics at Texas A\&M University during the fall of 1995 . He wishes to express his gratitude for financial assistance by the FRD of South Africa and for the hospitality bestowed upon him by Texas A\&M University.

\section{REFERENCES}

1. G. Bacella, The structure of QF-3 rings with zero singular ideal, Comm. Algebra 15 (1987), 1393-1446.

2. S. U. ChASE, A generalization of the ring of triangular matrices, Nagoya Math. J. 18 (1961), 13-25.

3. S. P. Coelho, The automorphism group of a structural matrix algebra, Linear Algebra Appl. 195 (1993), 35-58.

4. S. DÄSCÁlesCu and L. VAN WYK, Do isomorphic structural matrix rings have isomorphic graphs?, Proc. Amer. Math. Soc. 124 (1996), 1385-1391.

5. J. Fuelberth and J. Kuzmanovich, The structure of semiprimary and noetherian hereditary rings, Trans. Amer. Math. Soc. 212 (1975), 83-111.

6. R. GoRdon, Rings determined by $\mathcal{R}$-sets and a characterization of a class of semiperfect rings, Trans. Amer. Math. Soc. 155 (1971), 1-17.

7. M. HARADA, Hereditary semi-primary rings and tri-angular matrix rings, Nagoya Math. J. 17 (1960), 147-158.

8. T. W. Hungerford, Algebra (Springer-Verlag, New York, 1974).

9. N. JACOBSON, Structure of rings (Amer. Math. Soc. Colloq. Bull. XXXVII, Providence, R.I., 1964).

10. M. S. Li and J. M. Zelmanovitz, Artinian rings with restricted primeness conditions, J. Algebra 124 (1989), 139-148.

11. G. PILz, Near-rings, Revised Edition (North-Holland, Amsterdam, 1983). 
12. L. H. Rowen, Ring Theory, vol. I (Academic Press, New York, 1988).

13. K. C. SMITH, A ring associated with a near-ring, J. Algebra 182 (1996), 329-339.

14. K. C. SMITH and L. VAN WYK, An internal characterization of structural matrix rings, Comm. Algebra 22 (1994), 5599-5622.

15. L. VAN WYK, Matrix rings satisfying column sum conditions versus structural matrix rings, Linear Algebra Appl. 249 (1996), 15-28.

16. S. Veldsman, On the radicals of structural matrix rings, Monatsh. Math., to appear.

17. M. Weinstein, Examples of Groups (Polygonal Publishing House, Passaic, New Jersey, 1977).

Department of Mathematics

TEXAS A\&M UNIVERSITY

College Station

TEXAS 77843-3368

USA

E-mail address: ksmith@math.tamu.edu
Department of Mathematics

UNIVERSITY OF STELLENBosCh

Private Bag XI

Matieland 7602

SoUTH Africa

E-mail address:1vw@maties.sun.ac.za 